\title{
Uniqueness of positive solutions of nonlinear second order systems
}

Robert Dalmasso

Abstract. In this paper we discuss the uniqueness of positive solutions of the nonlinear second order system $-u^{\prime \prime}=g(v),-v^{\prime \prime}=f(u)$ in $(-R, R), u( \pm R)=v( \pm R)=0$ where $f$ and $g$ satisfy some appropriate conditions. Our result applies, in particular, to $g(v)=v, f(u)=u^{p}$, $p>1$, or $f(u)=\lambda u+a_{1} u^{p_{1}}+\cdots+a_{k} u^{p_{k}}$ with $p_{j}>1, a_{j}>0$ for $j=1, \ldots, k$ and $0 \leq \lambda<\mu_{1}^{2}$ where $\mu_{1}=\pi^{2} / 4 R^{2}$.

\section{Introduction.}

In this paper we discuss the uniqueness of positive solutions $(u, v) \in$ $\left(C^{2}[-R, R]\right)^{2}$ of the nonlinear second order system with homogeneous Dirichlet data

$$
\left\{\begin{aligned}
-u^{\prime \prime}(t) & =g(v(t)), & & -R<t<R, \\
-v^{\prime \prime}(t) & =f(u(t)), & & -R<t<R, \\
u( \pm R) & =v( \pm R)=0, & &
\end{aligned}\right.
$$

where $R>0$ is fixed and the functions $f, g \in C^{1}(\mathbb{R})$ satisfy the following assumptions

$$
\begin{aligned}
& 0<g(v) \leq v g^{\prime}(v), \quad \text { for } v>0 \\
& 0<f(u)<u f^{\prime}(u), \quad \text { for } u>0 .
\end{aligned}
$$


Of course $(u, v)>0$ means that $u>0$ and $v>0$ on $(-R, R)$.

It was proved by Troy [6] that $u$ and $v$ are symmetric about the origin and that $u^{\prime}<0$ and $v^{\prime}<0$ on $(0, R)$. It should be noted that in our situation the proof is considerably simpler. Moreover, by the Hopf boundary lemma [5, p. 4] here we also have $u^{\prime}(R)<0$ and $v^{\prime}(R)<0$. Therefore positive solutions of (1.1) can be treated as positive solutions of

$$
\begin{cases}-u^{\prime \prime}(t)=g(v(t)), & 0 \leq t<R \\ -v^{\prime \prime}(t)=f(u(t)), & 0 \leq t<R \\ u(R)=v(R)=u^{\prime}(0)=v^{\prime}(0)=0 . & \end{cases}
$$

The existence of positive solutions of nonlinear elliptic systems was examined by Clément, De Figueiredo and Mitidieri [1] in a bounded convex domain of $\mathbb{R}^{n}$ when $n \geq 2$ and by Peletier and Van Der Vorst [4] in a ball of $\mathbb{R}^{n}$ when $n \geq 4$. The question of the existence of positive solutions of problem (1.2) will be discussed in the last section of this paper.

Our main result is the following theorem.

Theorem 1.1. Let $f, g \in C^{1}(\mathbb{R})$ satisfy $\left(\mathrm{H}_{1}\right)$ and $\left(\mathrm{H}_{2}\right)$. Let $(u, v) \in$ $\left(C^{2}[-R, R]\right)^{2}$ be a positive solution of problem (1.1). Then $(u, v)$ is symmetric about the origin and is unique in the class of all positive solutions in $\left(C^{2}[-R, R]\right)^{2}$.

As a particular case of Theorem 1.1 we can state the following corollary concerning fourth order equations.

Corollary 1.1. Let $f \in C^{1}(\mathbb{R})$ satisfy $\left(\mathrm{H}_{2}\right)$. Let $u \in C^{4}[-R, R]$ be a positive solution of

$$
\left\{\begin{array}{l}
u^{(4)}(t)=f(u(t)), \quad-R<t<R \\
u( \pm R)=u^{\prime \prime}( \pm R)=0 .
\end{array}\right.
$$

Then $u$ is symmetric about the origin and is unique in the class of all positive solutions in $C^{4}[-R, R]$.

In our proofs we shall make an intensive use of the one dimensional maximum principle and the related Hopf boundary lemma [5], which we recall: 
Theorem A ([5, p. 2]). Suppose $u \in C^{2}(a, b) \cap C[a, b]$ satisfies the differential inequality

$$
u^{\prime \prime}+g(x) u^{\prime} \geq 0, \quad \text { for } a<x<b,
$$

with $g$ a bounded function. If $u \leq M$ in $(a, b)$ and if the maximum $M$ of $u$ is attained at an interior point of $(a, b)$, then $u \equiv M$.

Theorem B ([5, p. 4]). Suppose $u \in C^{2}(a, b) \cap C^{1}[a, b]$ is a nonconstant function which satisfies the differential inequality $u^{\prime \prime}+g(x) u^{\prime} \geq 0$ in $(a, b)$ and suppose $g$ is bounded on every closed subinterval of $(a, b)$. If the maximum of $u$ occurs at $x=a$ and $g$ is bounded below at $x=a$, then $u^{\prime}(a)<0$. If the maximum occurs at $x=b$ and $g$ is bounded above at $x=b$, then $u^{\prime}(b)>0$.

The outline of the paper is as follows. In Section 2 we introduce an initial value problem and we establish some preliminary results. Theorem 1.1 will be obtained as an immediate consequence of a crucial result that we state and prove in Section 3 (Theorem 3.1). Finally in Section 4 we prove an existence result and we give some examples to illustrate our theorem.

\section{Preliminary results.}

In order to prove our theorem we introduce the initial value problem

$$
\begin{cases}-u^{\prime \prime}(t)=g(v(t)), & t \geq 0 \\ -v^{\prime \prime}(t)=f(u(t)), & t \geq 0 \\ u(0)=\alpha, u^{\prime}(0)=0, & \\ v(0)=\beta, v^{\prime}(0)=0, & \end{cases}
$$

where $\alpha>0$ and $\beta>0$ are parameters. Throughout this section the functions $f, g \in C^{1}(\mathbb{R})$ are only assumed to be nondecreasing on $[0,+\infty)$ and such that $f(0)=g(0)=0, f(s), g(s)>0$ for $s>0$ and $\lim _{s \rightarrow+\infty} g(s)=+\infty$.

Below we prove some propositions which will be needed to state and prove our crucial result: Theorem 3.1. In the following proposition we establish the local existence and uniqueness of solutions of problem (2.1). 
Proposition 2.1. For any $\alpha>0, \beta>0$ there exists $T>0$ such that problem (2.1) on $[0, T]$ has a unique solution $(u, v) \in\left(C^{2}[0, T]\right)^{2}$.

Proof. Let $\alpha>0$ and $\beta>0$ be given. Choose $T>0$ such that

$$
T^{2} g(\beta) \leq \alpha \quad \text { and } \quad T^{2} f(\alpha) \leq \beta
$$

and consider the set of functions

$$
\begin{aligned}
Z=\left\{(u, v) \in(C[0, T])^{2}: \frac{\alpha}{2} \leq u(t) \leq \alpha\right. \text { and } & \frac{\beta}{2} \leq v(t) \leq \beta \\
& \text { for all } t \in[0, T]\}
\end{aligned}
$$

Clearly, $Z$ is a bounded closed convex subset of the Banach space $(C[0, T])^{2}$ endowed with the norm $\|(u, v)\|=\max \left\{\|u\|_{\infty},\|v\|_{\infty}\right\}$. Define

$$
L(u, v)(t)=\left(\alpha-\int_{0}^{t}(t-s) g(v(s)) d s, \beta-\int_{0}^{t}(t-s) f(u(s)) d s\right)
$$

for $t \in[0, T]$ and $(u, v) \in Z$. It is easily verified that $L$ is a compact operator mapping $Z$ into itself, and so there exists $(u, v) \in Z$ such that $(u, v)=L(u, v)$ by the Schauder fixed point theorem. Clearly $(u, v) \in\left(C^{2}[0, T]\right)^{2}$ and $(u, v)$ is a solution of $(2.1)$ on $[0, T]$. Since $f$ and $g$ are of class $C^{1}$ the uniqueness follows.

In view of Proposition 2.1, for any $\alpha, \beta>0$ problem (2.1) has a unique local solution: let $\left[0, T_{\alpha, \beta}\right)$ denote the maximum interval of existence of that solution $\left(T_{\alpha, \beta}=+\infty\right.$, possibly). Define

$$
P_{\alpha, \beta}=\left\{t \in\left(0, T_{\alpha, \beta}\right): u(\alpha, \beta, s) v(\alpha, \beta, s)>0, \text { for all } s \in[0, t]\right\}
$$

where $(u(\alpha, \beta, \cdot), v(\alpha, \beta, \cdot))$ is the solution of problem $(2.1)$ in $\left[0, T_{\alpha, \beta}\right)$. Clearly $P_{\alpha, \beta} \neq \varnothing$.

Proposition 2.2. For any $\alpha, \beta>0$ we have

$$
t_{\alpha, \beta}=\sup P_{\alpha, \beta}<T_{\alpha, \beta}
$$


Proof. If not, there exist $\alpha>0$ and $\beta>0$ such that $\sup P_{\alpha, \beta}=$ $T_{\alpha, \beta}$. Suppose first that $T_{\alpha, \beta}<+\infty$. Noting $u=u(\alpha, \beta, \cdot)$ and $v=v(\alpha, \beta, \cdot)$ we have

$$
\begin{aligned}
& 0 \leq u \leq \alpha \text { on }\left[0, T_{\alpha, \beta}\right) \\
& 0 \leq v \leq \beta \text { on }\left[0, T_{\alpha, \beta}\right)
\end{aligned}
$$

Since

$$
u^{\prime}(t)=-\int_{0}^{t} g(v(s)) d s \quad \text { and } \quad v^{\prime}(t)=-\int_{0}^{t} f(u(s)) d s
$$

for $t \in\left[0, T_{\alpha, \beta}\right)$, we conclude that $u, v, u^{\prime}$ and $v^{\prime}$ are bounded on $\left[0, T_{\alpha, \beta}\right)$ and we get a contradiction with the definition of $T_{\alpha, \beta}$. Now assume that $T_{\alpha, \beta}=+\infty$. Since $u^{\prime \prime}<0$ on $[0,+\infty)$ we deduce that

$$
u^{\prime}(t) \leq u^{\prime}(1)<0, \quad \text { for all } t \geq 1
$$

from which we get

$$
u(t) \leq u(1)+u^{\prime}(1)(t-1), \quad \text { for all } t \geq 1 .
$$

Thus we can find $t \geq 1$ such that $u(t)<0$ and we obtain a contradiction.

Proposition 2.3. For any $\alpha>0$ there exists a unique $\beta>0$ such that $u\left(\alpha, \beta, t_{\alpha, \beta}\right)=v\left(\alpha, \beta, t_{\alpha, \beta}\right)=0$.

Proof. We first prove the uniqueness. Let $\alpha>0$ be fixed. Suppose that there exist $\beta>\gamma>0$ such that $u\left(\alpha, \beta, t_{\alpha, \beta}\right)=v\left(\alpha, \beta, t_{\alpha, \beta}\right)=$ $u\left(\alpha, \gamma, t_{\alpha, \gamma}\right)=v\left(\alpha, \gamma, t_{\alpha, \gamma}\right)=0$. In order to simplify our notations, we denote $u(\alpha, \beta, t), v(\alpha, \beta, t), u(\alpha, \gamma, t)$ and $v(\alpha, \gamma, t)$ by $u(t), v(t)$, $w(t)$ and $z(t)$. Define $b=\min \left\{t_{\alpha, \beta}, t_{\alpha, \gamma}\right\}$. Suppose that there exists $a \in(0, b]$ such that $v-z>0$ on $[0, a)$ and $(v-z)(a)=0$. Since $u^{\prime \prime}-w^{\prime \prime}=g(z)-g(v)$ and $g$ is nondecreasing on $[0,+\infty)$, we deduce that $u^{\prime \prime}-w^{\prime \prime} \leq 0$ on $[0, a]$. Using the fact that $(u-w)(0)=(u-w)^{\prime}(0)=0$, Theorems A and B imply that $u-w \leq 0$ on $[0, a]$. Thus $v^{\prime \prime}-z^{\prime \prime}=$ $f(w)-f(u) \geq 0$ on $[0, a]$ since $f$ is nondecreasing on $[0,+\infty)$. We have $(v-z)(0)>0,(v-z)^{\prime}(0)=0$ and $(v-z)(a)=0$. Therefore Theorems $\mathrm{A}$ and $\mathrm{B}$ give a contradiction. Thus $v-z>0$ on $[0, b]$. As before we show that $u-w \leq 0$ on $[0, b]$. Since we have

$$
(v-z)(b)=\left\{\begin{array}{cl}
v\left(t_{\alpha, \gamma}\right)>0, & \text { if } t_{\alpha, \beta}>t_{\alpha, \gamma}, \\
0, & \text { if } t_{\alpha, \beta}=t_{\alpha, \gamma}, \\
-z\left(t_{\alpha, \beta}\right)<0, & \text { if } t_{\alpha, \beta}<t_{\alpha, \gamma},
\end{array}\right.
$$


necessarily $b=t_{\alpha, \gamma}<t_{\alpha, \beta}$. Now $(u-w)(b)=u\left(t_{\alpha, \gamma}\right)>0$ and we get a contradiction. The case $0<\beta<\gamma$ can be handled in the same way.

Now we prove the existence. Suppose that there exists $\alpha>0$ such that for any $\beta>0 u\left(\alpha, \beta, t_{\alpha, \beta}\right)>0$ or $v\left(\alpha, \beta, t_{\alpha, \beta}\right)>0$. Since $\alpha$ is fixed we shall write $u_{\beta}, v_{\beta}, t_{\beta}$ and $T_{\beta}$ instead of $u(\alpha, \beta, \cdot), v(\alpha, \beta, \cdot), t_{\alpha, \beta}$ and $T_{\alpha, \beta}$. Define the following two sets

$$
\begin{aligned}
& B=\left\{\beta>0: u_{\beta}\left(t_{\beta}\right)=0 \text { and } v_{\beta}\left(t_{\beta}\right)>0\right\}, \\
& C=\left\{\beta>0: u_{\beta}\left(t_{\beta}\right)>0 \text { and } v_{\beta}\left(t_{\beta}\right)=0\right\} .
\end{aligned}
$$

Then we have

$$
(0,+\infty)=B \cup C
$$

The proof of the proposition is completed by using the next lemma which contradicts (2.3).

Lemma 2.1. $B=C=\varnothing$.

The proof follows readily from (2.3) and the next two lemmas.

\section{Lemma 2.2.}

i) Suppose $B \neq \varnothing$. Then there exists $m>0$ such that $m \leq \inf B$.

ii) Suppose $C \neq \varnothing$. Then there exists $M>0$ such that $M \geq \sup C$.

Lemma 2.3. $B$ and $C$ are open.

Proof of Lemma 2.2. We have

$$
u_{\beta}(t)=\alpha-\int_{0}^{t}(t-s) g\left(v_{\beta}(s)\right) d s, \quad 0 \leq t<T_{\beta},
$$

and

$$
v_{\beta}(t)=\beta-\int_{0}^{t}(t-s) f\left(u_{\beta}(s)\right) d s, \quad 0 \leq t<T_{\beta} .
$$

i) Let $\beta \in B$. (2.2) and (2.4) imply

$$
t_{\beta} \geq\left(\frac{2 \alpha}{g(\beta)}\right)^{1 / 2}
$$


and from (2.5) we get

$$
\beta>\int_{0}^{t_{\beta}}\left(t_{\beta}-s\right) f\left(u_{\beta}(s)\right) d s
$$

Suppose that $\inf B=0$ and let $\left(\beta_{j}\right)$ be a sequence in $B$ decreasing to zero. Then $t_{\beta_{j}} \rightarrow+\infty$ by (2.6). From (2.7) we deduce that

$$
\beta_{j} \geq \int_{0}^{1}\left(t_{\beta_{j}}-s\right) f\left(u_{\beta_{j}}(s)\right) d s
$$

for $j$ large. Using (2.2) and (2.4) we have

$$
u_{\beta_{j}}(t) \geq \alpha-\frac{g\left(\beta_{j}\right)}{2} \geq \frac{\alpha}{2}
$$

for $t \in[0,1]$ and $j$ large. From (2.8) and (2.9) we get

$$
\beta_{j} \geq c
$$

for $j$ large where $c>0$ is independent of $j$. This gives a contradiction.

ii) Suppose that $\sup C=+\infty$ and let $\left(\beta_{j}\right)$ be a sequence in $C$ increasing to $+\infty$. By virtue of (2.2) we have

$$
0<u_{\beta_{j}}(t) \leq \alpha, \quad \text { for } t \in\left[0, t_{\beta_{j}}\right]
$$

(2.5) and (2.10) imply that $t_{\beta_{j}} \rightarrow+\infty$ as $j \rightarrow+\infty$. Then we can assume that $t_{\beta_{j}} \geq 1$ for all $j$ and that

$$
f(\alpha) \leq \beta_{j}, \quad \text { for all } j
$$

$(2.2),(2.5),(2.10)$ and (2.11) imply

$$
\frac{\beta_{j}}{2} \leq v_{\beta_{j}}(t) \leq \beta_{j}, \quad \text { for } t \in[0,1]
$$

and using (2.4) we deduce that $u_{\beta_{j}}(1) \leq \alpha-g\left(\beta_{j} / 2\right) / 2$. But then $u_{\beta_{j}}(1)<0$ for $j$ large contradicting $(2.10)$.

The proof of Lemma 2.3 depends on the following two lemmas. 


\section{Lemma 2.4.}

i) Suppose that $B \neq \varnothing$. Then for any $\beta \in B$ we have $u_{\beta}^{\prime}<0$ on $\left(0, t_{\beta}\right]$ and $v_{\beta}^{\prime}<0$ on $\left(0, t_{\beta}\right]$. If in addition $T_{\beta}<+\infty$, then for any $\gamma>\alpha$ (respectively, $\delta>\beta$ ) there exists $t \in\left(t_{\beta}, T_{\beta}\right)$ (respectively, $s \in$ $\left(t_{\beta}, T_{\beta}\right)$ ) such that $\left|u_{\beta}(t)\right|=\gamma$ and $\left|u_{\beta}(r)\right| \leq \gamma$ for $r \in[0, t]$ (respectively, $\left|v_{\beta}(s)\right|=\delta$ and $\left|v_{\beta}(r)\right| \leq \delta$ for $\left.r \in[0, s]\right)$.

ii) Suppose that $C \neq \varnothing$. Then for any $\beta \in C$ we have $u_{\beta}^{\prime}<0$ on $\left(0, t_{\beta}\right]$ and $v_{\beta}^{\prime}<0$ on $\left(0, t_{\beta}\right]$. If in addition $T_{\beta}<+\infty$, then for any $\gamma>\alpha$ (respectively, $\delta>\beta$ ) there exists $t \in\left(t_{\beta}, T_{\beta}\right)$ (respectively, $s \in$ $\left(t_{\beta}, T_{\beta}\right)$ ) such that $\left|u_{\beta}(t)\right|=\gamma$ and $\left|u_{\beta}(r)\right| \leq \gamma$ for $r \in[0, t]$ (respectively, $\left|v_{\beta}(s)\right|=\delta$ and $\left|v_{\beta}(r)\right| \leq \delta$ for $\left.r \in[0, s]\right)$.

Lemma 2.5. Suppose that $B \neq \varnothing$ and $C \neq \varnothing$. Then for any $\beta>0$ there exists $\eta>0$ such that $\min \left\{T_{\beta}, T_{\gamma}\right\}>\max \left\{t_{\beta}, t_{\gamma}\right\}$ for any $\gamma \in$ $(\beta-\eta, \beta+\eta)$.

Proof of Lemma 2.4. The first part of $\mathrm{i}$ ) is clear. Now assume that $T_{\beta}<+\infty$. If $u_{\beta}$ (respectively, $v_{\beta}$ ) is bounded on $\left[0, T_{\beta}\right.$ ), then $(2.5)$ (respectively, (2.4)) and (2.2) imply that $v_{\beta}$ (respectively, $u_{\beta}$ ), $u_{\beta}^{\prime}$ and $v_{\beta}^{\prime}$ are also bounded on $\left[0, T_{\beta}\right)$ contradicting the definition of $T_{\beta}$. Thus $u_{\beta}$ and $v_{\beta}$ can not be bounded on $\left[0, T_{\beta}\right)$ and the last part of i) follows easily. ii) can be proved similarly.

Proof of Lemma 2.5. Let $\beta$ be a fixed positive number. (2.3) implies that $\beta \in B \cup C$. Let $\gamma>0$. In the same way $\gamma \in B \cup C$. From (2.4), (2.5) using Gronwall's inequality we obtain

$$
\begin{aligned}
& \max \left\{\left|u_{\beta}(t)-u_{\gamma}(t)\right|,\left|v_{\beta}(t)-v_{\gamma}(t)\right|\right\} \\
& \leq|\beta-\gamma|\left(1+\int_{0}^{t} h(s) \exp \left(\int_{s}^{t} r h(r) d r\right) d s\right)
\end{aligned}
$$

for $t \in\left[0, \min \left\{T_{\beta}, T_{\gamma}\right\}\right.$, where the function $h$ is given by

$$
\begin{array}{r}
h(t)=\max \left\{\sup _{0 \leq \rho \leq 1}\left|f^{\prime}\left(\rho u_{\beta}(t)+(1-\rho) u_{\gamma}(t)\right)\right|,\right. \\
\left.\sup _{0 \leq \zeta \leq 1}\left|g^{\prime}\left(\zeta v_{\beta}(t)+(1-\zeta) v_{\gamma}(t)\right)\right|\right\}
\end{array}
$$

for $t \in\left[0, \min \left\{T_{\beta}, T_{\gamma}\right\}\right)$. Suppose that $\max \left\{t_{\beta}, t_{\gamma}\right\} \geq \min \left\{T_{\beta}, T_{\gamma}\right\}$. Then, by Proposition 2.2, $\min \left\{T_{\beta}, T_{\gamma}\right\}<+\infty$. If $\min \left\{T_{\beta}, T_{\gamma}\right\}=T_{\beta}$, 
then necessarily $\max \left\{t_{\beta}, t_{\gamma}\right\}=t_{\gamma}$. By Lemma 2.4 there exists $t \in$ $\left(t_{\beta}, T_{\beta}\right)$ such that $\left|u_{\beta}(t)\right|=2 \alpha$ and $\left|u_{\beta}(s)\right| \leq 2 \alpha$ for $s \in[0, t]$. Since $0 \leq u_{\gamma}(s) \leq \alpha$ and $0 \leq v_{\gamma}(s) \leq \gamma$ for $s \in[0, t]$ by Lemma 2.4, (2.12) and (2.13) imply

$$
\alpha \leq\left|u_{\beta}(t)-u_{\gamma}(t)\right| \leq c|\beta-\gamma|
$$

where $c>0$ depends on $\alpha, \beta, \gamma$ and $t \in\left(t_{\beta}, T_{\beta}\right)$; clearly $c$ is bounded with respect to $\gamma$ when $\gamma$ is in a bounded set. If $\min \left\{T_{\beta}, T_{\gamma}\right\}=T_{\gamma}$, then necessarily $\max \left\{t_{\beta}, t_{\gamma}\right\}=t_{\beta}$ and the proof is the same but now $t \in\left(t_{\gamma}, T_{\gamma}\right)$. Since in this case $T_{\gamma} \leq t_{\beta}$ we can choose in (2.14) the same $c$ as before. The lemma follows.

Proof of Lemma 2.3. 1) Suppose that $B$ is not open. (2.3) implies that there exists $\beta \in B$ and a sequence $\left\{\beta_{j}\right\}$ in $C$ such that $\beta_{j} \rightarrow \beta$ and $t_{\beta_{j}} \rightarrow T \in[0,+\infty]$. By Lemma 2.5 we can assume that $\min \left\{T_{\beta}, T_{\beta_{j}}\right\}>$ $\max \left\{t_{\beta}, t_{\beta_{j}}\right\}$ for all $j$ and so $T \leq T_{\beta}$. We first show that $T<+\infty$. If not, we can assume that $t_{\beta_{j}} \geq t_{\beta}$ for all $j$ by Proposition 2.2. Let $t \in\left[0, t_{\beta}\right]$. Using Lemma 2.4 we get

$$
\int_{0}^{t}(t-s) g\left(v_{\beta_{j}}(s)\right) d s \leq \frac{g\left(\beta_{j}\right) t^{2}}{2} .
$$

Choose $t \in\left(0, t_{\beta}\right]$ such that $g\left(\beta_{j}\right) t^{2} / 2 \leq \alpha / 2$ for all $j$. Then using again Lemma 2.4 and the fact that

$$
u_{\beta_{j}}(t)=\alpha-\int_{0}^{t}(t-s) g\left(v_{\beta_{j}}(s)\right) d s
$$

we obtain $u_{\beta_{j}}(s) \geq \alpha / 2$ for $s \in[0, t]$ and for all $j$. Since

$$
\begin{aligned}
\beta_{j} & =\int_{0}^{t_{\beta_{j}}}\left(t_{\beta_{j}}-s\right) f\left(u_{\beta_{j}}(s)\right) d s \\
& \geq \int_{0}^{t}\left(t_{\beta_{j}}-s\right) f\left(u_{\beta_{j}}(s)\right) d s \\
& \geq f\left(\frac{\alpha}{2}\right) t\left(t_{\beta_{j}}-\frac{t}{2}\right)
\end{aligned}
$$

for all $j$ we reach a contradiction. Now suppose that $T<T_{\beta}$. Then from (2.12), (2.13) and Lemma 2.4 we get

$$
\left|v_{\beta}\left(t_{\beta_{j}}\right)-v_{\beta_{j}}\left(t_{\beta_{j}}\right)\right| \leq c\left|\beta_{j}-\beta\right|, \quad \text { for all } j,
$$


where $c$ is a positive constant independent of $j$. Since $v_{\beta_{j}}\left(t_{\beta_{j}}\right)=0$ for all $j,(2.15)$ implies that $v_{\beta}(T)=0$. Therefore $T>t_{\beta}$. We can assume that $t_{\beta_{j}} \geq\left(T+t_{\beta}\right) / 2$ for all $j$. Let $t \in\left[t_{\beta}, T\right)$. Again we can assume that $t_{\beta_{j}} \geq t$ for all $j$. By Lemma 2.4 we have for all $j$

$$
0 \leq u_{\beta_{j}}(s) \leq \alpha, \quad \text { for } s \in[0, t]
$$

and

$$
0 \leq v_{\beta_{j}}(s) \leq \beta_{j}, \quad \text { for } s \in[0, t] .
$$

Then (2.12) and (2.13) give for $s \in[0, t]$

$$
\left|u_{\beta}(s)-u_{\beta_{j}}(s)\right| \leq c\left|\beta_{j}-\beta\right|, \quad \text { for all } j,
$$

where $c$ is a positive constant independent of $j$. Let $s=t_{\beta}$ in (2.16), we get

$$
u_{\beta_{j}}\left(t_{\beta}\right) \rightarrow 0 \quad \text { when } j \rightarrow+\infty .
$$

Since $u_{\beta_{j}}(t) \leq u_{\beta_{j}}\left(t_{\beta}\right)$ we obtain

$$
u_{\beta_{j}}(t) \rightarrow 0 \quad \text { when } j \rightarrow+\infty .
$$

From (2.16) with $s=t$ and (2.17) we deduce that $u_{\beta}(t)=0$. Since $t \in\left[t_{\beta}, T\right)$ is arbitrary we obtain a contradiction by using Lemma 2.4. Thus $T=T_{\beta}$. Then necessarily $T_{\beta}<+\infty$. By Lemma 2.4 we can find $s \in\left(t_{\beta}, T_{\beta}\right)$ such that $\left|v_{\beta}(s)\right|=2 \beta$ and $\left|v_{\beta}(r)\right| \leq 2 \beta$ for $r \in[0, s]$. We can assume that $t_{\beta_{j}} \geq s$ and $\beta / 2<\beta_{j}<3 \beta / 2$ for all $j$. Then from (2.12), (2.13) and Lemma 2.4 we obtain

$$
\frac{\beta}{2} \leq\left|v_{\beta}(s)-v_{\beta_{j}}(s)\right| \leq c\left|\beta_{j}-\beta\right|, \quad \text { for all } j,
$$

where $c$ is a positive constant independent of $j$. Clearly this is impossible.

2) Suppose that $C$ is not open. (2.3) implies that there exists $\beta \in C$ and a sequence $\left(\beta_{j}\right)$ in $B$ such that $\beta_{j} \rightarrow \beta$ and $t_{\beta_{j}} \rightarrow T \in[0,+\infty]$. By Lemma 2.5 we can assume that $\min \left\{T_{\beta}, T_{\beta_{j}}\right\}>\max \left\{t_{\beta}, t_{\beta_{j}}\right\}$ for all $j$ and so $T \leq T_{\beta}$. As in 1 ) we can show that $T<+\infty$. Now suppose that $T<T_{\beta}$. Then from (2.12), (2.13) and Lemma 2.4 we get

$$
\left|u_{\beta}\left(t_{\beta_{j}}\right)-u_{\beta_{j}}\left(t_{\beta_{j}}\right)\right| \leq c\left|\beta_{j}-\beta\right|, \quad \text { for all } j,
$$


where $c$ is a positive constant independent of $j$. Since $u_{\beta_{j}}\left(t_{\beta_{j}}\right)=0$ for all $j,(2.18)$ implies that $u_{\beta}(T)=0$. Therefore $T>t_{\beta}$. We can assume that $t_{\beta_{j}} \geq\left(T+t_{\beta}\right) / 2$ for all $j$. Let $t \in\left[t_{\beta}, T\right)$. Again we can assume that $t_{\beta_{j}} \geq t$ for all $j$. By Lemma 2.4 we have for all $j$

$$
0 \leq u_{\beta_{j}}(s) \leq \alpha, \quad \text { for } s \in[0, t]
$$

and

$$
0 \leq v_{\beta_{j}}(s) \leq \beta_{j}, \quad \text { for } s \in[0, t] .
$$

Then (2.12) and (2.13) give for $s \in[0, t]$

$$
\left|v_{\beta}(s)-v_{\beta_{j}}(s)\right| \leq c\left|\beta_{j}-\beta\right|, \quad \text { for all } j,
$$

where $c$ is a positive constant independent of $j$. Let $s=t_{\beta}$ in (2.19), we get

$$
v_{\beta_{j}}\left(t_{\beta}\right) \rightarrow 0 \quad \text { when } j \rightarrow+\infty
$$

Since $v_{\beta_{j}}(t) \leq v_{\beta_{j}}\left(t_{\beta}\right)$ we obtain

$$
v_{\beta_{j}}(t) \rightarrow 0 \quad \text { when } j \rightarrow+\infty \text {. }
$$

From (2.19) with $s=t$ and (2.20) we deduce that $v_{\beta}(t)=0$. Since $t \in\left[t_{\beta}, T\right)$ is arbitrary we obtain a contradiction by using Lemma 2.4. Thus $T=T_{\beta}$. Then necessarily $T_{\beta}<+\infty$. By Lemma 2.4 we can find $t \in\left(t_{\beta}, T_{\beta}\right)$ such that $\left|u_{\beta}(t)\right|=2 \alpha$ and $\left|u_{\beta}(r)\right| \leq 2 \alpha$ for $r \in[0, t]$. We can assume that $t_{\beta_{j}} \geq t$ for all $j$. Then from (2.12), (2.13) and Lemma 2.4 we obtain

$$
\alpha \leq\left|u_{\beta}(t)-u_{\beta_{j}}(t)\right| \leq c\left|\beta_{j}-\beta\right|, \quad \text { for all } j,
$$

where $c$ is a positive constant independent of $j$. Clearly this is impossible. The proof of the lemma is complete.

Now we introduce

$$
F(t)=\int_{0}^{t} f(s) d s \quad \text { and } \quad G(t)=\int_{0}^{t} g(s) d s .
$$

The following lemma will be needed in the next section.

Lemma 2.6. For any $\alpha>0, \beta>0$ we have

$(2.21) u^{\prime}(\alpha, \beta, t) v^{\prime}(\alpha, \beta, t)+F(u(\alpha, \beta, t))+G(v(\alpha, \beta, t))=F(\alpha)+G(\beta)$

for $t \in\left[0, T_{\alpha, \beta}\right)$.

The proof is obvious. 


\section{Proof of Theorem 1.1.}

We keep the notations introduced in Section 2. Clearly Theorem 1.1 is an immediate consequence of the following result.

Theorem 3.1. Let $f, g \in C^{1}(\mathbb{R})$ satisfy $\left(\mathrm{H}_{1}\right)$ and $\left(\mathrm{H}_{2}\right)$. Then for any $\alpha>0$ there exists a unique $(\beta(\alpha), t(\alpha)) \in(0,+\infty) \times(0,+\infty)$ such that $u(\alpha, \beta(\alpha), t(\alpha))=v(\alpha, \beta(\alpha), t(\alpha))=0$ and $u(\alpha, \beta(\alpha), t)>0$, $v(\alpha, \beta(\alpha), t)>0$ for $t \in[0, t(\alpha))$. Moreover $\beta(\alpha)$ is a strictly increasing function of $\alpha$ and $t(\alpha)$ is a strictly decreasing function of $\alpha$.

Proof. Let $\alpha>0$ be fixed. Since $f$ and $g$ verify the hypotheses used in Section 2 the existence and uniqueness of $(\beta(\alpha), t(\alpha))$ satisfying the conditions of the theorem are given by Proposition 2.3. Unfortunately the proof of the last part of the theorem is rather long. For $\alpha>0$, $\beta>0$ define

$$
\varphi(\alpha, \beta, t)=\frac{\partial u}{\partial \alpha}(\alpha, \beta, t), \quad \psi(\alpha, \beta, t)=\frac{\partial v}{\partial \alpha}(\alpha, \beta, t)
$$

and

$$
\rho(\alpha, \beta, t)=\frac{\partial u}{\partial \beta}(\alpha, \beta, t), \quad \chi(\alpha, \beta, t)=\frac{\partial v}{\partial \beta}(\alpha, \beta, t),
$$

for $t \in\left[0, T_{\alpha, \beta}\right)$. Then $\varphi, \psi, \rho$ and $\chi$ satisfy the linearized equations

$$
\begin{cases}-\varphi^{\prime \prime}(t)=g^{\prime}(v(t)) \psi(t), & 0 \leq t<T_{\alpha, \beta} \\ -\psi^{\prime \prime}(t)=f^{\prime}(u(t)) \varphi(t), & 0 \leq t<T_{\alpha, \beta} \\ \varphi(0)=1, \psi(0)=\varphi^{\prime}(0)=\psi^{\prime}(0)=0, & \end{cases}
$$

and

$$
\begin{cases}-\rho^{\prime \prime}(t)=g^{\prime}(v(t)) \chi(t), & 0 \leq t<T_{\alpha, \beta}, \\ -\chi^{\prime \prime}(t)=f^{\prime}(u(t)) \rho(t), & 0 \leq t<T_{\alpha, \beta}, \\ \chi(0)=1, \rho(0)=\rho^{\prime}(0)=\chi^{\prime}(0)=0 . & \end{cases}
$$

We first prove the following lemma.

Lemma 3.1. We have $\varphi^{\prime}>0$ (respectively, $\left.\chi^{\prime}>0\right)$ on $\left(0, t_{\alpha, \beta}\right]$ and $\psi^{\prime}<0$ (respectively, $\left.\rho^{\prime}<0\right)$ on $\left(0, t_{\alpha, \beta}\right]$. 
Proof. We have $\psi^{\prime \prime}(0)=-f^{\prime}(\alpha)<0$ (respectively, $\rho^{\prime \prime}(0)=-g^{\prime}(\beta)<$ 0 ). Then $\psi<0$ (respectively, $\rho<0)$ in $(0, \eta]$ for some $\eta>0$. Since the proof is the same in both cases we only prove that $\varphi^{\prime}>0$ and $\psi^{\prime}<0$ on $\left(0, t_{\alpha, \beta}\right]$. By what we have just seen we can define

$$
t_{0}=\sup \left\{t \in\left(0, t_{\alpha, \beta}\right]: \varphi \psi<0 \text { on }(0, t]\right\} .
$$

Since

$$
\varphi^{\prime}(t)=-\int_{0}^{t} g^{\prime}(v(s)) \psi(s) d s
$$

and

$$
\psi^{\prime}(t)=-\int_{0}^{t} f^{\prime}(u(s)) \varphi(s) d s
$$

we deduce that $\varphi^{\prime}>0$ and $\psi^{\prime}<0$ on $\left(0, t_{0}\right]$. Therefore $\varphi\left(t_{0}\right) \psi\left(t_{0}\right)<0$ and necessarily $t_{0}=t_{\alpha, \beta}$.

Now let $D=\left\{(\alpha, \beta, t): \alpha>0, \beta>0\right.$, and $\left.t \in\left[0, T_{\alpha, \beta}\right)\right\}$. It is well-known that $D$ is open in $(0,+\infty) \times(0,+\infty) \times[0,+\infty)$. Consider the map $H: D \longrightarrow \mathbb{R}^{2}$ defined by

$$
H(\alpha, \beta, t)=(u(\alpha, \beta, t), v(\alpha, \beta, t)) .
$$

Then $H \in C^{1}\left(D, \mathbb{R}^{2}\right)$ and

$$
H(\alpha, \beta(\alpha), t(\alpha))=0, \quad \text { for } \alpha>0 .
$$

Since by Theorems A and B we have

$$
u^{\prime}(\alpha, \beta(\alpha), t)<0 \quad \text { and } \quad v^{\prime}(\alpha, \beta(\alpha), t)<0
$$

for $t \in(0, t(\alpha)]$, using Lemma 3.1 we get

$$
\operatorname{det} D_{(\beta, t)} H(\alpha, \beta(\alpha), t(\alpha))=\left(\rho v^{\prime}-\chi u^{\prime}\right)(\alpha, \beta(\alpha), t(\alpha))>0 .
$$

Therefore by the implicit function theorem $\alpha \rightarrow(\beta(\alpha), t(\alpha))$ is a $C^{1}$ map for $\alpha>0$. Differentiating (3.3) with respect to $\alpha$ we get

$$
\begin{aligned}
\varphi(\alpha, \beta(\alpha), t(\alpha)) & +\rho(\alpha, \beta(\alpha), t(\alpha)) \beta^{\prime}(\alpha) \\
& +u^{\prime}(\alpha, \beta(\alpha), t(\alpha)) t^{\prime}(\alpha)=0
\end{aligned}
$$

and

$$
\begin{aligned}
\psi(\alpha, \beta(\alpha), t(\alpha)) & +\chi(\alpha, \beta(\alpha), t(\alpha)) \beta^{\prime}(\alpha) \\
& +v^{\prime}(\alpha, \beta(\alpha), t(\alpha)) t^{\prime}(\alpha)=0
\end{aligned}
$$


for $\alpha>0$. Since

$$
\beta^{\prime}(\alpha)=\left(\operatorname{det} D_{(\beta, t)} H(\alpha, \beta(\alpha), t(\alpha))\right)^{-1}\left(\psi u^{\prime}-\varphi v^{\prime}\right)(\alpha, \beta(\alpha), t(\alpha))
$$

we deduce from (3.4) and Lemma 3.1 that $\beta^{\prime}(\alpha)>0$. Define

$$
X(\alpha, t)=\varphi(\alpha, \beta(\alpha), t)+\rho(\alpha, \beta(\alpha), t) \beta^{\prime}(\alpha)
$$

and

$$
Y(\alpha, t)=\psi(\alpha, \beta(\alpha), t)+\chi(\alpha, \beta(\alpha), t) \beta^{\prime}(\alpha) .
$$

The proof of the theorem is completed by using (3.4), (3.5) and the next lemma.

Lemma 3.2. There exists $t_{0} \in(0, t(\alpha))$ (respectively, $\left.s_{0} \in(0, t(\alpha))\right)$ such that $X(\alpha, t)>0$ for $t \in\left[0, t_{0}\right)$ (respectively, $Y(\alpha, t)>0$ for $t \in$ $\left[0, s_{0}\right)$ ) and $X(\alpha, t)<0$ for $t \in\left(t_{0}, t(\alpha)\right]$ (respectively, $Y(\alpha, t)<0$ for $\left.t \in\left(s_{0}, t(\alpha)\right]\right)$.

Proof. In order to simplify our notations, we denote $X(\alpha, t), Y(\alpha, t)$, $u(\alpha, \beta(\alpha), t)$ and $v(\alpha, \beta(\alpha), t)$ by $X(t), Y(t), u(t)$ and $v(t)$. We have

$$
\left\{\begin{array}{l}
-X^{\prime \prime}(t)=g^{\prime}(v(t)) Y(t), \quad 0 \leq t<T_{\alpha, \beta(\alpha)} \\
-Y^{\prime \prime}(t)=f^{\prime}(u(t)) X(t), \quad 0 \leq t<T_{\alpha, \beta(\alpha)} \\
X(0)=1, X^{\prime}(0)=0, Y(0)=\beta^{\prime}(\alpha)>0, Y^{\prime}(0)=0 .
\end{array}\right.
$$

Lemma 3.3. $X \geq 0$ on $[0, t(\alpha)]$ if and only if $Y \geq 0$ on $[0, t(\alpha)]$.

Proof. Suppose that $X \geq 0$ on $[0, t(\alpha)]$. From (3.4), (3.5) and (3.6) we get $Y(t(\alpha)) \geq 0$. Then Theorem A implies that $Y \geq 0$ on $[0, t(\alpha)]$. The converse can be proved in the same way.

Now suppose that $X \geq 0$ on $[0, t(\alpha)]$. By Lemma 3.3 we also have $Y \geq 0$ on $[0, t(\alpha)]$. Then using $\left(\mathrm{H}_{1}\right),\left(\mathrm{H}_{2}\right)$ and $(3.4)$ we obtain

$$
\begin{aligned}
0 & <\int_{0}^{t(\alpha)}\left(f^{\prime}(u) u-f(u)\right) X=\int_{0}^{t(\alpha)} v^{\prime \prime} X-Y^{\prime \prime} u \\
& =\left(v^{\prime} X\right)(t(\alpha))+\left(u^{\prime} Y\right)(t(\alpha))+\int_{0}^{t(\alpha)} v X^{\prime \prime}-u^{\prime \prime} Y \\
& =\left(v^{\prime} X\right)(t(\alpha))+\left(u^{\prime} Y\right)(t(\alpha))+\int_{0}^{t(\alpha)}\left(g(v)-g^{\prime}(v) v\right) Y \leq 0
\end{aligned}
$$


and we reach a contradiction. In the same way $Y$ can not remain nonnegative on $[0, t(\alpha)]$. Thus we can define $t_{0}$ (respectively, $s_{0}$ ) to be the first zero of $X$ (respectively, $Y$ ) on $(0, t(\alpha))$. Moreover there exist $x \in\left(t_{0}, t(\alpha)\right)$ and $y \in\left(s_{0}, t(\alpha)\right)$ such that $X(x)<0$ and $Y(y)<0$. We shall prove that $X<0$ on $\left(t_{0}, t(\alpha)\right]$ and $Y<0$ on $\left(s_{0}, t(\alpha)\right]$ and this will complete the proof of Lemma 3.2. Suppose the contrary, then we have the following lemma.

Lemma 3.4. There exist $s_{1}, t_{1} \in\left(\max \left\{s_{0}, t_{0}\right\}, t(\alpha)\right]$ such that $X<0$ on $\left(t_{0}, t_{1}\right), X\left(t_{1}\right)=0, Y<0$ on $\left(s_{0}, s_{1}\right)$ and $Y\left(s_{1}\right)=0$. Moreover if $t=\min \left\{s_{1}, t_{1}\right\}$, then we have $X^{\prime}(t)>0$ and $Y^{\prime}(t)>0$.

Admitting the lemma for the moment, we show that we reach a contradiction. Differentiating (2.21) with respect to $\alpha$ and $\beta$ respectively and taking the value at $(\alpha, \beta(\alpha), t)$ with $t \in\left[0, T_{\alpha, \beta(\alpha)}\right)$ we get

$$
\varphi^{\prime} v^{\prime}+u^{\prime} \psi^{\prime}+g(v) \psi+f(u) \varphi=f(\alpha)
$$

and

$$
\rho^{\prime} v^{\prime}+u^{\prime} \chi^{\prime}+g(v) \chi+f(u) \rho=g(\beta(\alpha))
$$

for $t \in\left[0, T_{\alpha, \beta(\alpha)}\right)$, from which we deduce

$$
X^{\prime} v^{\prime}+Y^{\prime} u^{\prime}+g(v) Y+f(u) X=f(\alpha)+\beta^{\prime}(\alpha) g(\beta(\alpha))>0
$$

for $t \in\left[0, T_{\alpha, \beta(\alpha)}\right)$. Using (3.4), Lemma 3.4 and (3.8) for $t=\min \left\{s_{1}, t_{1}\right\}$ we see that the left hand side in (3.8) is negative and we get a contradiction.

In order to prove Lemma 3.4 we need

Lemma 3.5. $X(t)<0$ on $\left(t_{0}, t(\alpha)\right]$ if and only if $Y(t)<0$ on $\left(s_{0}, t(\alpha)\right]$.

Proof. Suppose that $X(t)<0$ on $\left(t_{0}, t(\alpha)\right]$. Then from (3.4), (3.5) and (3.6) we get $Y(t(\alpha))<0$. Suppose that $t_{0} \leq s_{0}$. Then Theorem A implies that $Y<0$ on $\left(s_{0}, t(\alpha)\right]$. Now if $t_{0}>s_{0}$, Theorems $\mathrm{A}$ and B imply that $Y^{\prime}<0$ on $\left(0, t_{0}\right]$. Thus $Y<0$ on $\left(s_{0}, t_{0}\right]$. Then using Theorem A we get $Y<0$ on $\left[t_{0}, t(\alpha)\right]$. The converse can be proved in the same way.

Proof of Lemma 3.4. Recall that our assumption is that $X$ can not remain negative on $\left(t_{0}, t(\alpha)\right]$ or that $Y$ can not remain negative on $\left(s_{0}, t(\alpha)\right]$. 
Case 1. $s_{0}=t_{0}$. By Theorems $\mathrm{A}$ and $\mathrm{B}$ we have $X^{\prime}\left(t_{0}\right)<$ 0 and $Y^{\prime}\left(t_{0}\right)<0$. Our assumption and Lemma 3.5 imply that there exist $s_{1}, t_{1} \in\left(t_{0}, t(\alpha)\right]$ such that $X<0$ on $\left(t_{0}, t_{1}\right), X\left(t_{1}\right)=0, Y<0$ on $\left(t_{0}, s_{1}\right)$ and $Y\left(s_{1}\right)=0$. If $s_{1}=t_{1}$ Theorems $\mathrm{A}$ and B imply that $X^{\prime}\left(t_{1}\right)>0$ and $Y^{\prime}\left(t_{1}\right)>0$. If $s_{1}>t_{1}$ Theorems A and B imply that $X^{\prime}>0$ on $\left[t_{1}, s_{1}\right]$. Therefore $Y^{\prime \prime}<0$ on $\left(t_{1}, s_{1}\right]$. Since $Y\left(t_{1}\right)<0$ and $Y\left(s_{1}\right)=0$ Theorems $\mathrm{A}$ and $\mathrm{B}$ imply that $Y^{\prime}\left(t_{1}\right)>0$. In the same way if $s_{1}<t_{1}$ we show that $X^{\prime}\left(s_{1}\right)>0$ and $Y^{\prime}\left(s_{1}\right)>0$.

Case 2. $s_{0}<t_{0}$. By Theorems A and B we have $Y^{\prime}<0$ on $\left(0, t_{0}\right]$. Our assumption and Lemma 3.5 imply that there exists $s_{1} \in\left(t_{0}, t(\alpha)\right]$ such that $Y<0$ on $\left(s_{0}, s_{1}\right)$ and $Y\left(s_{1}\right)=0$. Let $d \in\left(t_{0}, s_{1}\right)$ be such that $Y(d)=\min _{t_{0} \leq s \leq s_{1}} Y(s)$. Since $Y^{\prime \prime}(d)=-f^{\prime}(u(d)) X(d)$ we obtain $X(d) \leq 0$. Then Theorems A and B imply that $X^{\prime}\left(t_{0}\right)<0$. By virtue of Lemma 3.5 there exists $t_{1} \in\left(t_{0}, t(\alpha)\right]$ such that $X<0$ on $\left(t_{0}, t_{1}\right)$ and $X\left(t_{1}\right)=0$. Then we conclude as in Case 1 .

Case 3. $s_{0}>t_{0}$. The proof is analogous to that given in Case 2.

The proof is complete.

\section{An existence result and examples.}

We begin this section with an existence result concerning positive solutions of problem (1.2).

The method we use to prove the existence of a positive solution of problem (1.2) consists of first obtaining a priori estimates on the positive solutions and then applying well-known properties of compact mapping taking a cone in a Banach space into itself (see [3]).

We denote by $\mu_{1}$ the first eigenvalue of the operator $-d^{2} / d x^{2}$ on $(-R, R)$ with Dirichlet boundary conditions and $\varphi_{1}$ is the positive eigenfunction corresponding to $\mu_{1}\left(\mu_{1}=\pi^{2} / 4 R^{2}\right.$ and $\varphi_{1}(t)=C \cos (\pi t / 2 R)$ where $C>0$ is a constant).

Theorem 4.1. Let $f, g \in C(\mathbb{R})$ satisfy the following hypotheses

$$
f(s), g(s) \geq 0, \quad \text { for } s \geq 0,
$$

$\left(\mathrm{H}_{4}\right) \liminf _{s \rightarrow+\infty} \frac{f(s)}{s}>a>0, \liminf _{s \rightarrow+\infty} \frac{g(s)}{s}>b>0$ and $a b>\mu_{1}^{2}$,

$\left(\mathrm{H}_{5}\right) \quad \limsup _{s \rightarrow 0} \frac{f(s)}{s}<c, \quad \limsup _{s \rightarrow 0} \frac{g(s)}{s}<d \quad$ and $\quad c d<\mu_{1}^{2}$. 
Then problem (1.2) possesses at least one positive solution $(u, v) \in$ $\left(C^{2}[0, R]\right)^{2}$.

Proof. We first prove that there exists $M>0$ such that

$$
\|u\|_{\infty} \leq M \quad \text { and } \quad\|v\|_{\infty} \leq M
$$

for all positive solutions $(u, v) \in\left(C^{2}[0, R]\right)^{2}$ of (1.2). By $\left(\mathrm{H}_{4}\right)$, there exist $K_{j}>0$ for $j=1,2$ such that

$$
f(s) \geq a s-K_{1}, \quad \text { for } s \geq 0,
$$

and

$$
g(s) \geq b s-K_{2} \quad \text { for } s \geq 0 .
$$

Now let $(u, v) \in\left(C^{2}[0, R]\right)^{2}$ be a positive solution of (1.2). Then, $C$ denoting a generic positive constant, we have

$$
\begin{aligned}
\mu_{1}^{2} \int_{0}^{R} \varphi_{1} u d t & =-\mu_{1} \int_{0}^{R} u \varphi_{1}^{\prime \prime} d t=-\mu_{1} \int_{0}^{R} \varphi_{1} u^{\prime \prime} d t \\
& =\mu_{1} \int_{0}^{R} \varphi_{1} g(v) d t \geq b \mu_{1} \int_{0}^{R} \varphi_{1} v d t-C \\
& =-b \int_{0}^{R} v \varphi_{1}^{\prime \prime} d t-C=-b \int_{0}^{R} \varphi_{1} v^{\prime \prime} d t-C \\
& =b \int_{0}^{R} \varphi_{1} f(u) d t-C \geq a b \int_{0}^{R} \varphi_{1} u d t-C
\end{aligned}
$$

From (4.2) we deduce that

$$
\begin{gathered}
\int_{0}^{R} \varphi_{1} u d t \leq C, \quad \int_{0}^{R} \varphi_{1} v d t \leq C, \\
\int_{0}^{R} \varphi_{1} f(u) d t \leq C \quad \text { and } \quad \int_{0}^{R} \varphi_{1} g(v) d t \leq C,
\end{gathered}
$$

where $C$ is again a generic positive constant. Now we have

$$
u(t)=\int_{0}^{R} G(t, s) g(v(s)) d s \text { and } v(t)=\int_{0}^{R} G(t, s) f(u(s)) d s
$$


for $t \in[0, R]$, where $G(t, s)$ denotes the Green's function of the operator $-d^{2} / d x^{2}$ on $(-R, R)$ with Dirichlet boundary conditions. Since

$$
G(t, s)= \begin{cases}R-t, & 0 \leq s \leq t \leq R \\ R-s, & 0 \leq t \leq s \leq R\end{cases}
$$

we have

$$
0 \leq G(t, s) \leq R-s, \quad \text { for } \quad 0 \leq t, s \leq R
$$

We also have

$$
c_{1}(R-s) \leq \varphi_{1}(s) \leq c_{2}(R-s), \quad \text { for } \quad s \in[0, R],
$$

for some positive constants $c_{j}, j=1,2$. From (4.3)-(4.6) we easily get

$$
u(t) \leq C \quad \text { and } \quad v(t) \leq C \text { for } t \in[0, R],
$$

where $C$ is a positive constant and (4.1) is proved.

Now we can establish the existence of a positive solution of problem (1.2) by using Proposition 2.1 and Remark 2.1 of [3]. The arguments are by now well-known. However, in order that the paper be self contained, we provide details here (see [1], [2] or [4] for similar detailed proofs).

Let $\mathrm{X}$ denote the Banach space $(C[0, R])^{2}$ endowed with the norm $\|(u, v)\|=\max \left\{\|u\|_{\infty},\|v\|_{\infty}\right\}$. Define the cone

$$
C=\{(u, v) \in X:(u, v) \geq 0\} .
$$

For $((u, v), x) \in C \times[0,+\infty)$ we define

$$
F((u, v), x)(t)=\left(F_{1}((u, v), x)(t), F_{2}((u, v), x)(t)\right), \quad \text { for } t \in[0, R],
$$

where

$$
\begin{aligned}
& F_{1}((u, v), x)(t)=\int_{0}^{R} G(t, s) g(v(s)+x) d s \\
& F_{2}((u, v), x)(t)=\int_{0}^{R} G(t, s) f(u(s)+x) d s
\end{aligned}
$$

and

$$
\Phi(u, v)=F((u, v), 0)
$$


By $\left(\mathrm{H}_{3}\right), F$ maps $C \times[0,+\infty)$ into $C$. Since $G$ is continuous, it is wellknown that $F$ is compact. $\left(\mathrm{H}_{3}\right)$ and $\left(\mathrm{H}_{5}\right)$ imply that $f(0)=g(0)=0$, hence $\Phi(0)=0$. Now the following properties hold:

i) $(u, v) \neq \theta \Phi(u, v)$ for all $\theta \in[0,1]$ and $(u, v) \in C$ such that $\|(u, v)\|=r$ for sufficiently small $r>0$. Indeed by $\left(\mathrm{H}_{5}\right)$ we can choose $r>0$ such that $f(s) \leq c s$ and $g(s) \leq d s$ for $0 \leq s \leq r$. Now suppose that there exist $\theta \in[0,1]$ and $(u, v) \in C$ such that $(u, v)=\theta \Phi(u, v)$ with $\|(u, v)\|=r$. Then

$$
\left\{\begin{array}{l}
-u^{\prime \prime}(t)=\theta g(v(t)), \quad 0 \leq t<R \\
-v^{\prime \prime}(t)=\theta f(u(t)), \quad 0 \leq t<R \\
u(R)=v(R)=u^{\prime}(0)=v^{\prime}(0)=0
\end{array}\right.
$$

By Theorem A, $u, v>0$ on $[0, R)$. We have

$$
\begin{aligned}
\mu_{1}^{2} \int_{0}^{R} \varphi_{1} u d t & =-\mu_{1} \int_{0}^{R} u \varphi_{1}^{\prime \prime} d t=-\mu_{1} \int_{0}^{R} \varphi_{1} u^{\prime \prime} d t \\
& =\mu_{1} \theta \int_{0}^{R} \varphi_{1} g(v) d t \leq d \mu_{1} \int_{0}^{R} \varphi_{1} v d t \\
& =-d \int_{0}^{R} v \varphi_{1}^{\prime \prime} d t=-d \int_{0}^{R} \varphi_{1} v^{\prime \prime} d t \\
& =d \theta \int_{0}^{R} \varphi_{1} f(u) d t \leq c d \int_{0}^{R} \varphi_{1} u d t
\end{aligned}
$$

and we reach a contradiction because the integrals are nonzero.

ii) By $\left(\mathrm{H}_{4}\right)$, there exists $x_{0}>0$ such that

$$
f(s+x) \geq a(s+x) \geq a s
$$

and

$$
g(s+x) \geq b(s+x) \geq b s, \quad \text { for } s \geq 0, x \geq x_{0}>0 .
$$

Then using the same arguments as in the proof of (4.1) and Theorem A, we can show that $F((u, v), x) \neq(u, v)$ for all $(u, v) \in C$ and $x \geq x_{0}$.

iii) Now we note that the constant in (4.1) can be chosen independently of the parameter $x \in\left[0, x_{0}\right]$ for each fixed $x_{0} \in(0,+\infty)$ if we consider positive solutions of (1.2) for the family of nonlinearities 
$f_{x}(t)=f(t+x), g_{x}(t)=g(t+x), t \geq 0$. Thus we can find a constant $R>r$ such that $F((u, v), x) \neq(u, v)$ for all $x \in\left[0, x_{0}\right]$ and $(u, v) \in C$ with $\|(u, v)\|=R$.

Thus we may apply Proposition 2.1 and Remark 2.1 stated in [3] to conclude that $\Phi$ has a nontrivial fixed point $(u, v) \in C$. Theorem A and the properties of the Green's function imply that any nontrivial fixed point of $\Phi$ in $C$ yields a positive solution of $(1.2)$ in $\left(C^{2}[0, R]\right)^{2}$. The proof of the theorem is complete.

REMARK. Note that, for the a priori estimates, condition $\left(\mathrm{H}_{3}\right)$ is not needed. We need it merely to insure that the maps $\Phi$ and $F$ are conepreserving.

We conclude this section with some examples to which our theorems apply.

a) We first consider problem (1.3) where $g(v)=v$. When $f(u)=$ $\sum_{j=1}^{k} a_{j} u^{p_{j}}$ for $u>0$ with $p_{j}>1$ and $a_{j}>0$ for $j=1, \ldots, k$ and $k \geq 1$ or $f(u)=u^{r} /\left(1+u^{s}\right)$ for $u>0$ with $r-1>s>0$, Theorem 4.1 implies the existence of a positive solution of (1.3) and Corollary 1.1 gives the uniqueness.

b) For problem (1.1) we can take $f$ as in a) and $g$ of the same type as $f$. Then the existence of a positive solution of (1.1) follows from Theorem 4.1 and the uniqueness is given by Theorem 1.1.

c) Take

$$
f(u)=\lambda u+u^{p} \quad \text { and } \quad g(v)=\mu v+v^{q}, \quad u, v>0,
$$

with $p, q>1, \lambda, \mu>0$ and $\lambda \mu<\mu_{1}^{2}$. By Theorem 4.1 there exists a positive solution of (1.1). Then Theorem 1.1 gives the uniqueness.

This is an example of a perturbed linear system. Consider the linear eigenvalue problem

$$
\begin{cases}-u^{\prime \prime}=\lambda_{2} v, & \text { in }(-R, R), \\ -v^{\prime \prime}=\lambda_{1} u, & \text { in }(-R, R), \\ u>0, v>0, & \text { in }(-R, R), \\ u( \pm R)=v( \pm R)=0 . & \end{cases}
$$

The next lemma is a particular case of a result proved by Van Der Vorst [7] (see also [2] for an extension of this result). 
Lemma 4.1. Problem (4.7) has a solution if and only if

$$
\lambda_{j}>0, \text { for } j=1,2, \quad \text { and } \quad \lambda_{1} \lambda_{2}=\mu_{1}^{2} .
$$

The solution is given by $u=c_{1} \varphi_{1}, v=c_{2} \varphi_{1}$ where $c_{1}>0$ is an arbitrary constant and $c_{2}=c_{1}\left(\lambda_{1} / \lambda_{2}\right)^{1 / 2}$.

Clearly the above lemma shows that conditions $\left(\mathrm{H}_{1}\right)$ and $\left(\mathrm{H}_{2}\right)$ are sharp.

\section{References.}

[1] Clément, P. H., De Figueiredo, D. and Mitidieri, E., Positive solutions of semilinear elliptic systems. Comm. Partial Diff. Equations 17 (1992), 923-940.

[2] Dalmasso, R., Positive solutions of nonlinear elliptic systems. Ann. Polonici Math. LVIII (1993), 201-212.

[3] De Figueiredo, D., Lions, P. L. and Nussbaum, R. D., A priori estimates and existence of positive solutions of semilinear elliptic equations. $J$. Math. Pures et Appl. 61 (1982), 41-63.

[4] Peletier, L. A. and Van Der Vorst, R. C. A. M., Existence and nonexistence of positive solutions of nonlinear elliptic systems and the biharmonic equation. J. Diff. Int. Equations 5 (1992), 747-767.

[5] Protter, M. and Weinberger, H., Maximum principles in differential equations. Prentice Hall, 1967.

[6] Troy, W. C., Symmetry properties in systems of semilinear elliptic equations. J. Differential Equations 42 (1981), 400-413.

[7] Van Der Vorst, R. C. A. M., Variational identities and applications to differential systems. Arch. Rational Mech. Anal. 116 (1991), 375-398.

Recibido: 10 de julio de 1.993

Robert Dalmasso

Laboratoire LMC-IMAG

Equipe EDP-Tour IRMA-B.P. 53

F-38041 Grenoble Cedex 9, FRANCE

Robert.Dalmasso@imag.fr 